\title{
Oncology
}

Adenocarcinoma, gastric 422

Adipokine 26

Adiponectin 26, 261

Advanced disease 228, 298

Anaplastic glioma 21

Anastrozole 415

Angiogenesis 246

Angiogenic inhibitor 246

Anthracycline resistance 407

Arhgdib 430

Azoxymethane 252

Barrett's esophagus 281

Basic fibroblast growth factor 246

Biliary tract cancers 311

Biochemotherapy 33

Biomarker 9

Biopsy 366

Bombesin 340

Bone marrow 192

- sarcomas 58

Brain metastases 273

Breast cancer 136, 145, 154, 210, 228, 376, 415

Capecitabine 21, 169, 221, 228, 298

Carboplatin 198, 407, 419

Carcinoembryonic antigen 52

Carcinogenesis 357

Case-control study 305

Cdx2 357

Cervical cancer 340

- carcinoma 273

Cervix cancer 145,154

Cetuximab 185

Chemoradiation 169

Chemoradiotherapy 316

Chemoresistance 81

Chemosensitivity, in vitro 104

Chemotherapy 185, 221, 311, 335

Cholangiocarcinoma 127

Cisplatin 76, 104, 426

Claudin-2 357

Colon cancer 52, 98

Colorectal cancer 72, 185, 298, 395

- carcinoma 9

Combination chemotherapy 298

Compliance 145, 154

CPT-11 65

Cyclin D1 112

Cyclophosphamide 210

CYR61 118
Definitive chemoradiotherapy 328

Diagnosis 273, 366

Disease progression 401

Disheveled 112

DNA methylation 346

Docetaxel chemotherapy 2

Drug resistance 21

Drug-related toxicity 204

Dual-modality PET/CT 316

E1AF 384

Elderly 290

- patients 228

Endobronchial metastases 395

Endoscopy 395

EphB1 238

Epidermal growth factor receptor 104, 185,281

Erythema multiforme 127

Esophageal cancer 346, 366

- neoplasms 281

Estrogen receptor-alpha 81

5-Fluorouracil 104, 270

Fructosamine 3 kinase 72

Ganglioside 430

Gastric cancer 2, 65, 192, 215

- carcinoma 118, 238

Gastrin-releasing peptide 340

- - receptor 340

Gastrointestinal stromal tumor 324

Gemcitabine 41, 130, 221, 290, 311, 407, 426

Gemzar $^{\circledR} 130$

Gene expression 72

Glioblastoma 21

Glycation 72

Glypican3 389

GM3 430

Head and neck cancer 198

- _ _ squamous cell carcinoma 104

Heat shock 98

Hepatocellular carcinoma 90

HER-2 104

High-dose chemotherapy 58

Hodgkin's lymphoma 422

Hormonal therapy 305

Hyperthermia 98

Imatinib mesylate 324

Immunohistochemical staining 238
Immunohistochemistry 81, 340, 357, 389

Insulin resistance 261

Interferon- $\boldsymbol{\gamma}$-inducible protein 10246

Interleukin-2 33

Intrahepatic large vessels 90

Invasive ductal carcinoma 112

Irinotecan 9, 65, 76, 185

Leptin 26

Lipoplatin 426

Liver metastasis 324

Locally advanced non-small cell lung cancer 316

Low-dose chemotherapy 21

Lung cancer 261, 305

- carcinoids 162

Lymph node metastasis 401

Lymphocytes 215

Lymphoma, cutaneous T cell 130

-, large cell 130

-, non-Hodgkin 130

Macrophage infiltration 316

Malignancy 127

Matrilysin 384

Matrix metalloproteinase 252

Melanoma 33, 401

Mesothelioma 426

Metastasis 192

Metastatic breast cancer 407

Microarray 252, 366

MMP-7 118

mTOR 430

Multikinase inhibitor 204

Multistage tissue 357

Mycosis fungoides 130

Myelodysplastic syndromes 26

Neoadjuvant 316

Neoplasms 41

Neutrophil/lymphocyte ratio 215

Neutrophils 215

Obesity 261

Oesophageal cancer 328

Older 210

Oral squamous cell carcinoma 198

Ovarian cancer 136,177

Oxaliplatin 270, 311

p27 $7^{\text {kip } 1} 52$

Paclitaxel 198 
Pancreas 41

Pancreatic cancer 221, 335

Paraneoplastic dermatosis 127

Pegylated liposomal doxorubicin 210

Pemetrexed 9

Peritoneal metastasis 136

Phase II trial 228

PhIP 252

Pioglitazone 21

Portal vein tumor thrombosis 90

Predictive factors 185

Preoperative 366

Prognosis 41

Prognostic factor 81, 215, 335

Pulmonary fibrosis 270

- neuroendocrine tumours 162

\section{Radiotherapy 90}

Randomised controlled trial 145, 154

Raptor 430

Rat colon carcinogenesis 252

Real-time RT-PCR 104, 384

Receptors 261

Rectal cancer 52, 169, 384

Renal cell cancer 204

Retinoid receptor-alpha 81

- receptor-beta 81
Rictor 430

Rofecoxib 21

RT-PCR 252

-, quantitative real-time 238

RUNX 346

S-1 65

Sclerosing glomerulonephritis 415

Screening 145, 154

Second-line chemotherapy 335, 426

Serotonin 118

Serous ovarian cancer 81

Simultaneous preoperative chemoradiotherapy 198

Smad 346

Small-cell lung cancer 76

Soft tissue sarcomas 58

Somatostatin analogues 162

Sorafenib 204

Stage I seminoma 419

Stem cell support 58

Survival 273

-, long-term disease-free 33

-, overall 33

Temozolomide 21

Thiazolidinedione 261
Thoracoscopy 426

Three-week schedule 76

Thyroid cancer 389

Topotecan 177

Toxicity 270

Treatment 273

Tumor necrosis factor- $\alpha 430$

Unfit 290

Urothelial cancer 290

Uterine endometrial cancer 246

Vinorelbine 228, 426

WASF 376

WASP 376

- verprolin homologues 376

WAVE 376

Western blot 81

Wnt/ $\beta$-catenin pathway 112 